\title{
L'Encyclopédie « entière » ou comment mettre en relation le discours et les planches, à travers l'exemple du traitement en image et en texte de l'étonnement
}

Véronique Le $\mathrm{Ru}$

CIRLEP EA 4299

Université de Reims Champagne-Ardenne

C'est un fait, on lit souvent l'Encyclopédie en ne tenant compte que des dix-sept volumes de texte, mais on peut tenter de penser l'articulation des dix-sept volumes de discours et des onze volumes de planches. Il est vrai que l'histoire de l'édition de l'Encyclopédie autorise à séparer le texte et les planches. En effet, si l'édition des dix-sept volumes de discours a été entrecoupée (un volume par an paraît entre 1751 et 1757 puis les dix derniers volumes paraissent en 1765), celle des planches s'opère sans interruption de 1762 à 1772 . Entre 1745 et 1780 (date à laquelle tous les suppléments et tables sont parus), l'ouvrage a grossi démesurément : de cinq volumes dont un de cent vingt planches prévus initialement en mars 1745 , on est passé à la fin de la même année à dix volumes dont deux de six cents planches, pour aboutir aux dix-sept volumes de discours et onze volumes de planches auxquels il faut ajouter quatre volumes de supplément de texte, un volume de supplément de planches et deux volumes de tables. Pour comprendre le lien entre le texte et les planches, il est éclairant de se reporter au supplément de planches et, particulièrement, à l'Avertissement de ce volume :

On ne doit point être surpris que l'Explication, dont nous accompagnons les Planches de ce Supplément, soit si courte, tandis qu'elle est si longue dans quelques volumes des Planches de l'Encyclopédie. C'est que toutes les figures de nos Planches sont suffisamment expliquées dans les articles auxquels elles se rapportent, de sorte qu'une simple indication suffit ici, pour éviter des répétitions superflues; au lieu que dans l'Encyclopédie les Planches ont été ajoutées au texte où souvent même elles ne sont pas annoncées, de manière qu'il fallait nécessairement les accompagner d'une explication assez étendue pour les rendre intelligibles, et suppléer par là le texte (1777).

Les Suppléments de l'Encyclopédie ont un statut à part. Premièrement, l'éditeur ou plutôt le groupe d'éditeurs a changé : à Briasson, David, Le Breton et Durand succèdent, pour les sept volumes de suppléments et de tables, Rey à Amsterdam et, à Paris, Panckoucke (auquel se joignent Stoupe et Brunet pour le supplément de planches). Deuxièmement, l'Avertissement au Supplément de planches nous apprend que les articles des quatre volumes de supplément de texte annoncent et expliquent les 
planches regroupées dans le cinquième volume de supplément, ce qui n'était pas le cas pour l'Encyclopédie. L'Avertissement, a contrario, nous instruit du fait que, dans l'Encyclopédie, les dix-sept volumes de texte sont relativement coupés des onze volumes de planches puisque souvent les planches se rapportant à tel ou tel article n'y sont même pas annoncées. Les onze volumes de planches fonctionnent, par conséquent, de manière plutôt autonome avec leur Explication qui vient «suppléer par là le texte ». Cela pourrait conduire, indique la suite de l'Avertissement, à séparer le texte des planches voire à considérer les planches comme inutiles : «Il n'en faut pas conclure, comme ont fait quelques gens ou peu éclairés ou mal intentionnés, que ces planches sont inutiles » (ibid.). Au contraire, l'Encyclopédie sans les planches est un ouvrage mutilé : «Si l'on sépare cette vaste et riche collection de planches en onze volumes, des dix-sept volumes de discours, on n'aura qu'un ouvrage mutilé et imparfait» (ibid.). Diderot, lui aussi, insiste dans le Prospectus in Discours préliminaire de l'Encyclopédie ${ }^{1}$ (1984 : 144-149) et dans l'article ENCYCLOPÉDIE (1755 t. V : 641, 647) sur l'intérêt des figures et des planches dans la description des arts.

Il serait présomptueux de vouloir mettre au jour l'interaction des volumes de texte et des volumes de planches tant les uns comme les autres sont matière à méditer et à rêver ${ }^{2}$. En revanche, il peut être utile et pertinent de proposer un exemple de mise en relation du discours et des planches. L'exemple que nous avons choisi de développer est le traitement en texte et en image d'une passion, à savoir l'étonnement. C'est dans la planche XXIV de DESSEIN [DESSIN] dans le troisième volume de Planches paru en 1763 qu'on trouve non pas directement l'étonnement mais la figure 2 de l'admiration avec étonnement, qui suit la figure 1 de l'admiration simple. Mais avant d'en venir à la représentation de l'étonnement dans l'Encyclopédie, il importe de relier la notion à son histoire dans la mesure où les trois planches XXIV, XXV et XXVI de DESSEIN où l'on trouve l'expression des passions sont faites d'après le peintre Le Brun qui luimême s'inspire, dans sa représentation, des Passions de l'âme de Descartes (nous pré- sentons en annexe à la fin de l'article les trois planches relatives aux passions).

Le terme étonnement vient du latin extonare ou attonare qui

\footnotetext{
${ }^{1}$ Titre abrégé par la suite en D.P.E

${ }^{2}$ L'Encyclopédie, en ce sens, ne cesse de se renouveler dans les lectures et les rêveries que le texte aussi bien que les planches suscitent. Barthes, dans son texte intitulé «Les Planches de l'Encyclopédie » (1972: 89-105), note qu'elles ne constituent pas un système fermé. L'image encyclopédique n'est pas repliée sur elle-même mais déployée, actualisée dans sa puissance d'informations, et s'ouvre ainsi à toute lecture future. En cela l'image est véritablement encyclopédique : elle met en cycle la représentation du savoir. On pourrait ajouter que ce que Barthes dit de l'image vaut aussi pour le texte.
} 
signifie « frapper du tonnerre » d'où le sens vieilli de commotion violente ou d'ébranlement et d'où le sens technique de lézarde dans un édifice (étonner une voûte signifie la lézarder ou étonner un diamant signifie le fêler). De là découle le sens moral de choc ou d'ébranlement voire d'épouvante ou de terreur. Dans la langue classique qui nous intéresse ici, le terme étonnement désigne une violente émotion ou une stupeur à la vue d'un spectacle extraordinaire avant de signifier plus généralement une surprise causée par quelque chose d'inattendu. Descartes, dans l'article 73 de la deuxième partie des Passions de l'âme, définit l'étonnement comme un excès d'admiration qui ne peut être que mauvais. Pourquoi ? Parce que l'étonnement tel qu'il est physiologiquement décrit empêche tout mouvement, bloque la pensée sur ce qui impressionne et interdit la connaissance de ce qui étonne : être étonné pour Descartes c'est être frappé de stupeur. Quand il y a excès d'admiration, le mouvement d'attention vers l'objet rare engendré par l'admiration ${ }^{3}$ n'a pas lieu, on est figé dans l'impression de l'objet qui étonne et les esprits animaux sont tous tendus vers l'endroit du cerveau où est cette impression pour la conserver : « ce qui fait que tout le corps demeure immobile comme une statue, et qu'on ne peut apercevoir de l'objet que la première face qui s'est présentée, ni par conséquent en acquérir une plus particulière connaissance » (Descartes, article 73, partie II des Passions de l'âme in tome XI, p. 383 des Euvres, Paris, Adam et Tannery en 11 tomes, Paris, 1897-1909; rééd. par les éditions Vrin et CNRS, 1964-1974; 1996) ${ }^{4}$.

On le constate, le sens classique a déplacé l'origine de l'étonnement de l'oreille à l'œil. Le terme tel qu'il est employé par Descartes ne se rapporte ni au tonnerre ni à l'étonnement de la Renaissance, qui désignait, d'après Littré, un genre de bruit d'oreilles fait d'une grande commotion ou secousse du cerveau ${ }^{5}$, mais se rapporte explicitement à la vue. Être étonné c'est être frappé de stupeur par ce qu'on voit et non par ce qu'on entend. Être étonné c'est même plus précisément être immobile et avoir le regard figé ou fixé sur la seule face de l'objet qui s'est présentée à la vue. Mais que ce soit par l'oreille ou par l'œil qu'il advienne, l'étonnement a d'abord un sens négatif, il pèche par excès : c'est une fêlure du comportement qui empêche toute réaction ou toute

\footnotetext{
${ }^{3}$ Descartes précise, dans l'article 71 des Passions de l'âme, que l'admiration a pour unique objet la connaissance de la chose qu'elle admire.

${ }^{4}$ Titre abrégé par la suite en Euvres, suivi du tome en chiffres romains et de la page en chiffres arabes.

${ }^{5}$ Diderot, dans l'article ART de l'Encyclopédie, mentionne ce sens auditif de l'étonnement chez Montaigne selon lequel «les armes à feu sont de si peu d'effet, sauf l'étonnement des oreilles » (1751 t. I : 717).
} 
connaissance.

Ce sens négatif tend à s'atténuer au XVIII ${ }^{\mathrm{e}}$ siècle : l'étonnement n'est pas directement mauvais ou négatif mais il apparaît plutôt comme le point de rupture dans la gradation des passions et sépare ce qui est positif de ce qui est négatif. Dans l'articulation des passions qui ont toutes entre elles un air de famille lié à la surprise, on constate que l'étonnement se situe toujours au point de tension du positif vers le négatif.

Dans l'article SURPRISE de l'Encyclopédie, Diderot commence par rappeler l'unité originelle des émotions : «Je ne sais s'il y a beaucoup de diversité dans la manière dont nos organes sont émus. Tout se réduit peut-être aux différents degrés d'intensité et à la différence des objets » $(1765 \mathrm{t}$. XV : 693). Puis il représente, à un extrême, la figure de l'émotion la plus légère de plaisir et, à l'autre extrême, la figure de la terreur :

depuis l'émotion la plus légère de plaisir, celle qui altère à peine les traits de notre visage, qui n'émeut que l'extrémité de nos lèvres et y répand la finesse du souris, et qui n'ajoute qu'une nuance imperceptible d'éclat à celui de nos yeux : jusqu'aux agitations, aux transports de la terreur qui nous tient la bouche entr'ouverte, le front pâle, le visage transi, les yeux hagards, les cheveux hérissés, tous les membres convulsés et tremblants, ce n'est peut-être qu'un accroissement de termes (ibid.).

L'Encyclopédie contient trois planches de l'expression des passions d'après Le Brun : on trouve dans la planche XXIV la figure 1 de l'Admiration simple qui pourrait correspondre à l'émotion la plus légère de plaisir décrite par Diderot. Voici, dans l'explication qui accompagne les planches, la description de cette première figure d'après Le Brun :

Cette passion ne causant que peu d'agitation, n'altère que très peu les parties du visage; cependant le sourcil s'élève, l'œil s'ouvre également un peu plus qu'à l'ordinaire. La prunelle placée également entre les paupières, paraît fixée vers l'objet, la bouche s'entr'ouvre et ne forme pas de changement marqué dans les joues (1763 t. III : 11).

La filiation entre Les Passions de l'âme de Descartes et les figures de Le Brun est manifeste : pas de changement marqué dans les joues, Le Brun dessine ce que Descartes dit de l'admiration, à savoir que « cette passion a cela de particulier qu'on ne remarque point qu'elle soit accompagnée d'aucun changement qui arrive dans le cœur et dans le sang, ainsi que les autres passions » (article 71, partie II des Passions de l'âme in Cuvres, XI, 381). De même que Descartes fait succéder à l'admiration (articles 70 à 72) l'étonnement (article 73), de même Le Brun fait succéder à la figure de l'Admiration simple celle de l'Admiration avec étonnement décrite ainsi :

Les mouvements qui accompagnent cette passion, ne sont presque différents de 
ceux de l'admiration simple, qu'en ce qu'ils sont plus vifs et plus marqués, les sourcils plus élevés, les yeux plus ouverts, la prunelle plus éloignée de la paupière inférieure et plus fixe, la bouche plus ouverte, et toutes les parties dans une tension plus sensible (1763 t. III : 11).

La prunelle est fixe, Descartes disait que sous le coup de l'étonnement « on ne peut apercevoir de l'objet que la première face qui s'est présentée » (article 73, partie II des Passions de l'âme in Euvres, XI, 383).

Pour ce qui concerne la figure qui est à l'autre extrême, la terreur, décrite par Diderot dans l'article SURPRISE, il est difficile de deviner à quelle représentation il fait référence vu qu'aucune planche de Le Brun ne comporte de figure de la terreur, absence du reste toujours conforme aux Passions de l'âme où aucun article ne traite de la terreur. Mais ce qui nous intéresse davantage dans l'article de Diderot, c'est la gradation des passions liées à la surprise qu'il propose en conclusion et la situation de pivot entre le positif et le négatif qu'y tient l'étonnement: surprise, admiration, étonnement, alarme, frayeur, terreur (nous soulignons le terme). L'étonnement, dans la gradation des passions, est l'instance affective où le plaisir bascule vers la peine ${ }^{6}$.

Cette analyse du statut de limite de l'étonnement est confirmée par l'article PASSIONS de l'Encyclopédie qui est anonyme. L'esprit de l'article est cartésien comme l'atteste la définition initiale des passions :

Les penchants, les inclinations, les désirs et les aversions, poussés à un certain degré de vivacité, joints à une sensation confuse de plaisir ou de douleur, occasionnés ou accompagnés de quelque mouvement irrégulier du sang et des esprits animaux, c'est ce que nous nommons passions (1765 t. XII : 142).

L'esprit cartésien se remarque également dans la classification des passions proposée dans la deuxième partie de l'article. On y lit en effet que «L'admiration est la première et la plus simple de nos passions, elle mérite à peine ce nom ; c'est ce sentiment vif et subit de plaisir qui s'excite chez nous à la vue d'un objet dont la perfection nous frappe » (ibid.: 144). Suit une comparaison de l'admiration avec l'étonnement qui vient confirmer son statut de limite entre le plaisir et la peine : "On pourrait lui opposer l'étonnement, si ce mot n'était restreint à exprimer un pareil sentiment de peine qui naît à la vue d'une difformité peu commune, et l'horreur en particulier que cause la vue d'un vice ou d'un crime extraordinaire » (ibid.). Le mot admiration exprime un sentiment vif et subit de plaisir tandis que le mot étonnement est restreint à l'expression d'un

\footnotetext{
${ }^{6}$ De même dans l'article STYLE DE MOTET in tome XV (1765 : 556) de l'Encyclopédie, on lit que ce style fleuri et varié est propre à remuer les passions, mais surtout à exciter l'admiration, l'étonnement, la douleur. On retrouve dans cette gradation des passions la même situation de pivot de l'étonnement.
} 
« pareil sentiment de peine », c'est-à-dire d'un sentiment également vif et subit mais de peine.

L'article précise l'origine physique ou morale de l'étonnement : le sentiment vif et subit de peine est un sentiment du monstrueux causé par la vue d'une difformité physique ou morale (« sentiment de peine qui naît à la vue d'une difformité peu commune, et l'horreur en particulier que cause la vue d'un vice ou d'un crime »[ibid.]). Or ce qui peut être dit monstrueux, c'est ce qui provoque chez nous, êtres humains et vivants, un écart morphologique ou moral par rapport à nos représentations habituelles et à nos attentes de l'humain et du vivant. Comme l'explique si bien Canguilhem dans son texte intitulé «La monstruosité et le monstrueux », le sentiment du monstrueux naît d'un raté par rapport à notre confiance dans l'ordre que nous a enseigné la vie : «Il suffit d'une déception de cette confiance, d'un écart morphologique, d'une apparence d'équivocité spécifique pour qu'une crainte radicale s'empare de nous » (Canguilhem, La monstruosité et le monstrueux in La connaissance de la vie, 1965 : 171). Cette crainte causée par le monstrueux est radicale, elle touche à nos racines d'être humain et vivant mais en même temps elle est ambivalente car le monstrueux n'est pas le monstre, comme le précise Canguilhem, et si la vie est pauvre en monstres, elle est riche en monstrueux parce que le monstrueux est un monde. Le sentiment du monstrueux, l'étonnement est ambivalent parce qu'il est «Crainte [...] et même terreur panique, d'une part. Mais aussi, d'autre part, curiosité, et jusqu'à la fascination. Le monstrueux est du merveilleux à rebours, mais c'est du merveilleux malgré tout » (ibid. : 173).

Le monstrueux ne s'inscrit pas dans le registre du monde gouverné par les lois de la raison où le monstre, comme le fou, est d'abord montré puis enfermé, mais il s'inscrit dans le registre d'un antimonde, dans le chaos des exceptions sans lois. Au terme de son étude, Canguilhem identifie cet antimonde au monde imaginaire, trouble et vertigineux du monstrueux (ibid.: 184). On comprend alors, par la référence de l'étonnement à la difformité physique ou morale proposée dans l'article PASSIONS, pourquoi l'étonnement a un statut de limite entre le plaisir et la peine et pourquoi il peut être considéré comme un point de rupture dans la gradation des passions entre ce qui est positif et ce qui est négatif.

Cet article PASSIONS, par la corrélation qu'il établit entre l'étonnement et l'horreur, nous apprend aussi dans quel autre sens l'étonnement peut être conçu comme un point de rupture. Rappelons que Descartes, dans l'article 85 des Passions de l'âme (in Euvres, XI, 392), définit deux sortes de haine, celle qui se rapporte aux choses mauvaises et 
celle qui se rapporte aux choses laides qu'il propose d'appeler horreur ou aversion pour la distinguer de la haine d'ordre moral. Si celle-ci est tributaire d'un jugement moral sur ce qui est convenable ou contraire à notre nature, l'horreur est tributaire d'un jugement esthétique sur ce qui est convenable ou contraire à notre goût. Descartes indique ainsi la voie d'un traitement esthétique des passions, du moins de l'agrément et de l'horreur. Or il avait préalablement dans l'article 73 condamné l'étonnement parce qu'il était stérile sur le plan épistémologique (assimilable à la stupeur, l'étonnement empêchait toute connaissance, voir article 73, partie II des Passions de l'âme in Euvres, XI, 383). La conception de l'étonnement et des passions développée dans l'Encyclopédie confirme ces deux points : on renonce à lire l'étonnement et les passions en général dans une perspective épistémologique, on lui préfère une perspective esthétique. Autrement dit, on ne reproche plus à l'étonnement sa stérilité en connaissances parce qu'on lui donne sens non pas dans une épistémologie mais dans une esthétique. L'étonnement s'avère ainsi être le point de rupture entre passions négatives et passions positives mais également le point de rupture entre deux lectures épistémologique et esthétique des passions.

Si l'on revient à l'article SURPRISE de l'Encyclopédie et à l'opposition que Diderot instaure entre le plaisir et la terreur, on note que le sens de la vue et la représentation dominent dans la description qu'il fait de l'émotion la plus légère, description qu'on a pu, en ce sens, rapprocher de la figure 1 de l'admiration simple de la planche XXIV d'après Le Brun. Quant à la terreur décrite également de manière très picturale par Diderot (voir article SURPRISE de l'Encyclopédie in 1765 t. XV : 693 : « la terreur qui nous tient la bouche entr'ouverte, le front pâle, le visage transi, les yeux hagards, les cheveux hérissés, tous les membres convulsés et tremblants »), on ne peut lui faire correspondre aucune figure de Le Brun mais on peut, pour l'éclairer, consulter l'article TERREUR de Jaucourt. Or ce qui frappe dans cet article, c'est le parti pris qui s'y exprime, à savoir le traitement esthétique de la terreur, ce qui n'est pas le cas d'autres articles de l'Encyclopédie où il est question de la terreur.

En effet, les articles au titre composé comme ALARMETERREUR-EFFROI (article de Diderot in 1751 t. I : 277-278), EFFRAYANT-EFFROYABLE-TERRIBLE (article de d'Alembert in 1755 t. V : 412) ou encore EFFRAYÉ-ÉPOUVANTÉ-ALARMÉ (article également rédigé par d'Alembert in 1755 t. V : 412) proposent une gradation des passions liées à la peur, gradation où la terreur conçue comme le paroxysme de l'effroi occupe un extrême :

L'effroi et la terreur naissent l'un et l'autre d'un grand danger : mais la terreur peut être panique et l'effroi ne l'est jamais. Il semble que l'effroi soit dans les 
organes, et que la terreur soit dans l'âme. La terreur a saisi les esprits ; les sens sont glacés d'effroi ; un prodige répand la terreur ; la tempête glace d'effroi (voir l'article ALARME, TERREUR, EFFROI de Diderot, 1751 t. I : 277).

Cependant, dans l'article TERREUR, le traitement est tout autre, Jaucourt n'instaure aucune classification des passions mais insiste sur le sens esthétique de la terreur. La définition initiale du terme, par la référence au récit, laisse déjà la place à la représentation de la terreur : «grand effroi causé par la présence ou par le récit de quelque grande catastrophe » (1765 t. XVI : 184). Suit un commentaire où apparaît très vite le traitement esthétique de la terreur que Jaucourt réfléchit dans une perspective théâtrale et, plus précisément, tragique :

la terreur $[\ldots]$ semble $[\ldots]$ consister dans la totalité des incidents, qui en produisant chacun leur effet, et menant insensiblement l'action à sa fin, opèrent sur nous cette appréhension salutaire, qui met un frein à nos passions sur le triste exemple d'autrui, et nous empêche par là de tomber dans ces mêmes malheurs, dont la représentation nous arrache des larmes (ibid.).

Jaucourt insiste ici sur la fonction cathartique de la représentation de la terreur et les termes qu'il utilise (produire son effet, mener l'action à sa fin) nous installent d'emblée devant une scène de théâtre «dont la représentation nous arrache des larmes ». Ceci est confirmé dans la suite de l'article : «On ne peut trop appuyer sur les beautés de ce qu'on appelle terreur dans le tragique. C'est pourquoi nous ne pouvons manquer d'avoir une grande opinion de la tragédie des anciens : l'unique objet de leurs poètes était de produire la terreur et la pitié » (ibid.). Toute la fin de l'article développe des exemples de représentation de la terreur dans le théâtre moderne afin de montrer, sous la mention implicite de la querelle des Anciens et des Modernes, qu'il n'est pas moins méritoire que l'ancien.

Du XVII ${ }^{\mathrm{e}}$ au XVIII ${ }^{\mathrm{e}}$ siècle, on passe ainsi d'un déni de l'étonnement comme stupeur de l'entendement ou, en d'autres termes, comme obstacle épistémologique, à sa valorisation comme objet d'une esthétique. Or l'étonnement est d'autant plus valorisé au sein d'une lecture esthétique des passions, qu'il n'est pas une passion ordinaire mais qu'il a le statut de pivot entre les principes de plaisir et de peine et qu'il fonde en cela une nouvelle esthétique. Ceci est manifeste dans la partie de l'article GOÛT rédigée par Montesquieu. L'article GOÛT est en réalité un recueil de textes sur le goût : on a d'abord la partie physiologique rédigée par Jaucourt, puis un texte de Voltaire, puis un texte de Montesquieu et enfin un texte de d'Alembert. Le texte de Montesquieu posthume et inachevé ${ }^{7}$, constitue la

\footnotetext{
${ }^{7}$ Voici l'introduction de d'Alembert à l'Essai sur le goût de Montesquieu qui suit le texte de Voltaire dans l'article GOÛT : « Nous joindrons à cet excellent article, le fragment sur le goût, que M. le président de Montesquieu destinait à l'Encyclopédie [...] ; ce fragment a été
} 
deuxième partie de l'article GOÛT intitulée Essai sur le goût dans les choses de la nature et de l'art. Montesquieu commence par distinguer le beau d'avec le bon avant d'élaborer sa définition du goût. Contrairement à Voltaire et à d'Alembert qui insistent, dans leur conception du goût, sur la nécessité de compléter le sentiment par le discernement, il considère que le sentiment est le principe du goût (Diderot partage ce point de vue). Par conséquent, l'analyse des sources du plaisir, même si elle est intéressante dans une perspective philosophique, ne peut rien apporter au jugement du goût :

On croit d'abord qu'il suffirait de connaître les diverses sources de nos plaisirs, pour avoir le goût, et que quand on a lu ce que la philosophie nous dit là-dessus, on a du goût, et que l'on peut hardiment juger des ouvrages. Mais le goût naturel n'est pas une connaissance de théorie ; c'est une application prompte et exquise des règles même que l'on ne connaît pas. Il n'est pas nécessaire de savoir que le plaisir que nous donne une certaine chose que nous trouvons belle, vient de la surprise ; il suffit qu'elle nous surprenne autant qu'elle le doit, ni plus, ni moins (1757 t. VII : 762).

Il est vrai que Montesquieu sépare le goût naturel du goût acquis qui tolère les préceptes. Mais pour lui, le goût est avant tout affaire de sentiment : « La définition la plus générale du goût, sans considérer s'il est bon ou mauvais, juste ou non, est ce qui nous attache à une chose par le sentiment » (ibid.).

Cette définition l'amène à développer une analyse de la surprise, sentiment fondateur du goût. Dans un paragraphe intitulé Des plaisirs de la surprise, il note que l'âme goûte tous les plaisirs qui viennent de la surprise: «sentiment qui plaît à l'âme par le spectacle et par la promptitude de l'action, car elle aperçoit ou sent une chose qu'elle n'attend pas, ou d'une manière qu'elle n'attendait pas » (ibid.: 764). Dans un paragraphe

trouvé imparfait dans ses papiers : l'auteur n'a pas eu le temps d'y mettre la dernière main ; mais les premières pensées des grands maîtres méritent d'être conservées à la postérité, comme les esquisses des grands peintres » (1757 t. VII : 761-762, le texte de Montesquieu occupe les pages 762 à 767). Effectivement, dans la lettre à d'Alembert du 16 novembre 1753, Montesquieu lui fait part à la fois de son refus d'écrire les articles DÉMOCRATIE et DESPOTISME et de son projet d'écrire l'article GOÛT. L'Essai sur le goût est publié pour la première fois dans l'article GOÛT du tome VII de l'Encyclopédie (1757) puis en 1758 dans les Euvres complètes (Amsterdam et Leipsick. Arkstée et Merkus. t. III. 611-638). Il est repris dans l'édition des Euvres posthumes (Londres. 137-200) avec quelques variantes et dans celle de 1798 qui lui ajoute le chapitre «des Règles »sous-titré «chapitre qui termine l'Essai sur le goût ». Ce chapitre et les suivants avaient été communiqués au baron Walckenaer par Charles Roullet, président à la cour de Bordeaux qui les publie en 1804 dans les Annales littéraires de l'Europe (t. II. 301-311). Le texte de l'édition de 1758 avec les variantes de l'édition des Euvres posthumes de 1783 et les compléments publiés par Walckenaer sont regroupés dans le texte de l'Essai sur le goût annoté et postfacé par L. Desgraves (1993). 
ultérieur intitulé Progression de la surprise, Montesquieu explique que ce qui fait les grandes beautés ce n'est pas simplement la surprise mais le fait qu'elle dure et augmente: «Ce qui fait les grandes beautés, c'est lorsqu'une chose est telle que la surprise est d'abord médiocre, qu'elle se soutient, augmente, et nous mène ensuite à l'admiration » (ibid. : 766). Mais pour passer de la surprise à l'admiration, l'instance affective décisive est l'étonnement comme l'atteste l'exemple de grande beauté développée par Montesquieu, à savoir l'église Saint-Pierre de Rome : "L'exacte proportion de la fameuse église de Saint-Pierre, fait qu'elle ne paraît pas d'abord aussi grande qu'elle est [...] Mais à mesure que l'on examine, l'œil la voit s'agrandir, l'étonnement augmente » (ibid.). À vrai dire, l'étonnement est ce qui produit un certain embarras de l'âme, une fêlure entre ce qu'elle voit et ce qu'elle a vu, fêlure qui est le signe même du jugement du beau. À propos du dôme de Saint-Pierre, Montesquieu écrit :

on sait que Michel-Ange voyant le panthéon, qui était le plus grand temple de Rome, dit qu'il en voulait faire un pareil, mais qu'il voulait le mettre en l'air. Il fit donc sur ce modèle le dôme de Saint-Pierre : mais il fit les piliers si massifs, que ce dôme qui est comme une montagne que l'on a sur la tête, paraît léger à l'œil qui le considère. L'âme reste donc incertaine sur ce qu'elle voit et ce qu'elle sait, et elle reste surprise de voir une masse en même temps si énorme et si légère (ibid.).

Montesquieu considère l'étonnement qui produit cet embarras de l'âme si important qu'il lui consacre toute la fin de son analyse du goût. Cette partie finale, intitulée «Des beautés qui résultent d'un certain embarras de l'âme », érige l'étonnement en fondement du jugement de goût :

Souvent la surprise vient à l'âme de ce qu'elle ne peut pas concilier ce qu'elle voit avec ce qu'elle a vu. Il y a en Italie un grand lac, qu'on appelle le lac majeur; c'est une petite mer dont les bords ne montrent rien que de sauvage. À quinze milles dans le lac sont deux îles d'un quart de mille de tour, qu'on appelle les Borromées, qui est à mon avis le séjour du monde le plus enchanté. L'âme est étonnée de ce contraste romanesque, de rappeler avec plaisir les merveilles des romans, où après avoir passé par des rochers et des pays arides, on se trouve dans un lieu fait pour les fées (ibid.).

L'étonnement est ainsi conçu comme ce qui fait goûter véritablement le beau car la surprise est certes un principe de plaisir mais qui pèche par défaut. Pour qu'il y ait jugement du beau, il faut que la surprise augmente, qu'elle devienne étonnement. Celui-ci est promu en norme du goût. Par un effet de chiasme, l'étonnement change de statut dans l'Encyclopédie: si, pour Descartes, c'était l'admiration qui était la norme par rapport à laquelle l'étonnement péchait par excès, empêchant toute connaissance; pour Montesquieu comme pour Diderot $^{8}, c^{\prime}$ est l'étonnement qui est la norme par rapport à laquelle la surprise

\footnotetext{
${ }^{8}$ Voir son article ÉTONNEMENT de l'Encyclopédie où il fait de l'étonnement l'instance
} 
pèche par défaut, empêchant l'admiration et tout jugement du beau. Mais il est vrai que l'effet de chiasme est rendu possible par le changement de point de vue : on n'étudie plus les passions en physicien mais en esthéticien.

normative par rapport à la surprise, qui est un étonnement dégénéré : "Selon la nature de l'événement, l'étonnement dégénère en surprise » (1756t. VI : 68). 


\section{ANNEXE DES PLANCHES}

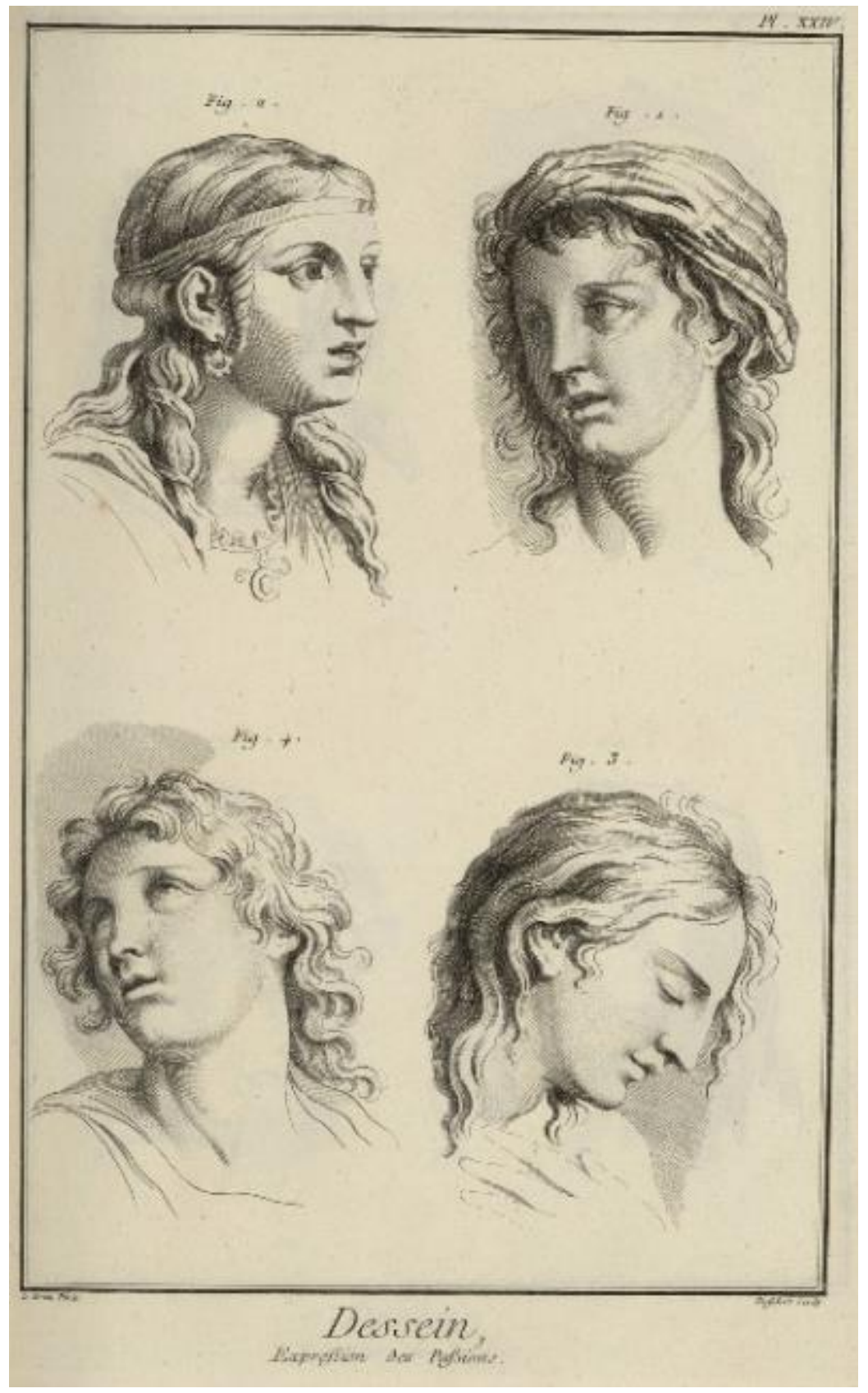

L'Encyclopédie. Volume 24. Planches 3, 1763, Planche XXIV, Bibliothèque Mazarine, $2^{\circ} 3442$ Volume 24, https://mazarinum.bibliotheque-mazarine.fr/idurl/1/2135 


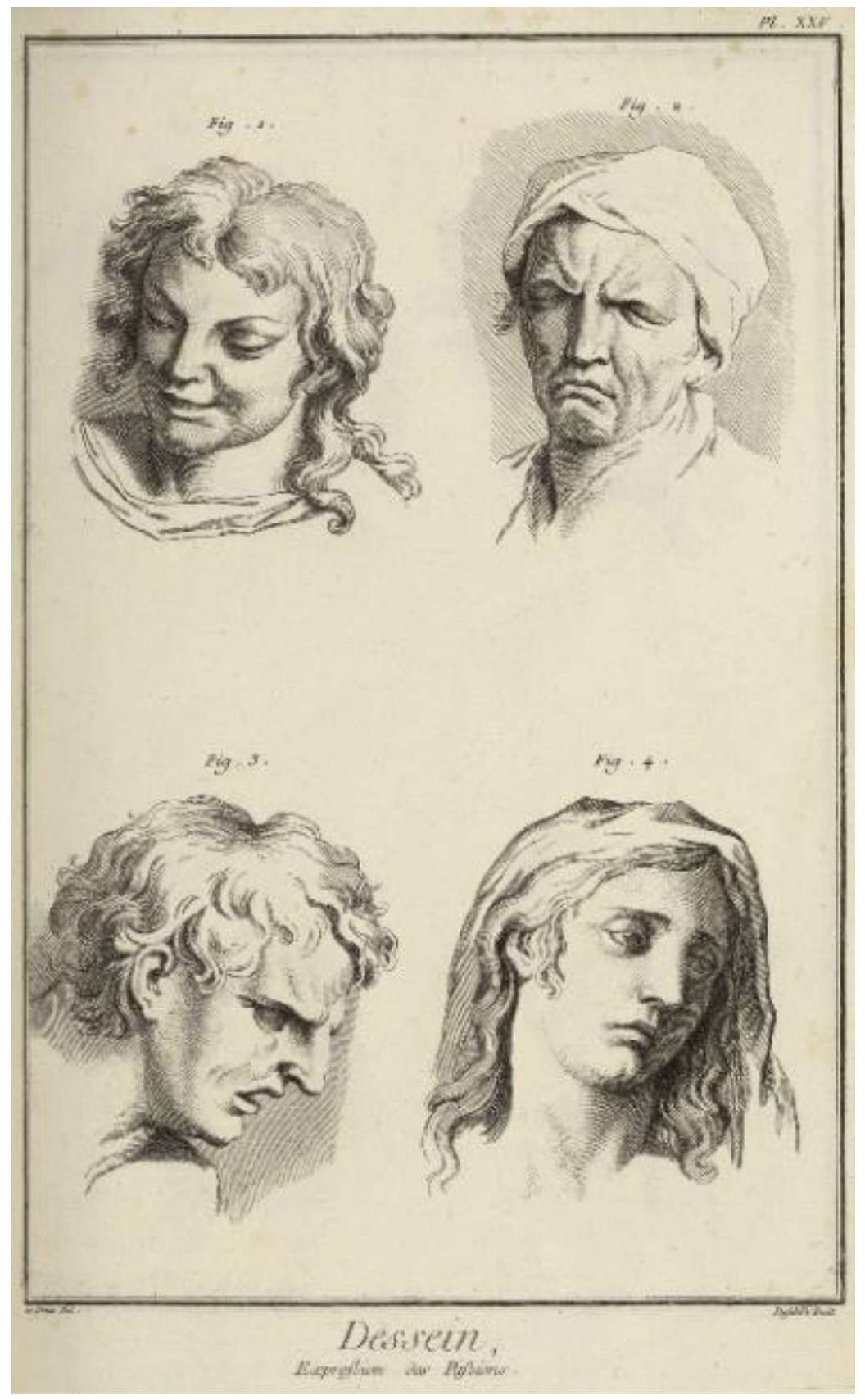

L'Encyclopédie. Volume 24. Planches 3, 1763, Planche XXV, Bibliothèque Mazarine, $2^{\circ} 3442$ Volume 24, https://mazarinum.bibliotheque-mazarine.fr/idurl/1/2135 


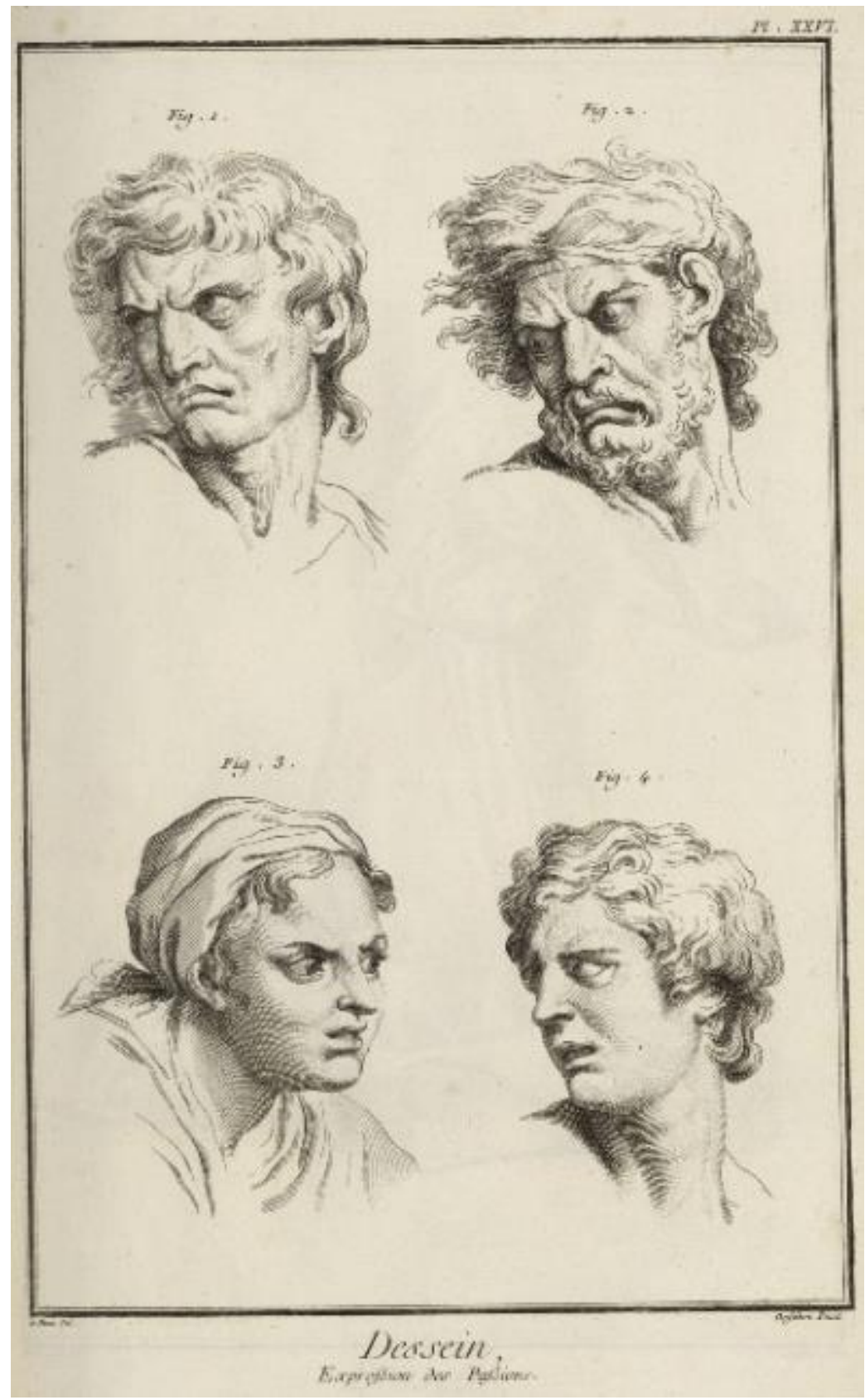

L'Encyclopédie. Volume 24. Planches 3, 1763, Planche XXVI, Bibliothèque Mazarine, $2^{\circ} 3442$ Volume 24, https://mazarinum.bibliotheque-mazarine.fr/idurl/1/2135 


\section{Bibliographie}

1777. Avertissement de la Suite du Recueil de Planches sur les sciences, les arts libéraux et les arts méchaniques avec leur explication. $5^{\mathrm{e}}$ volume de Supplément. Paris : Panckoucke, Stoupe, Brunet.

D’Alembert J. 1984. Discours préliminaire de l'Encyclopédie contenant aussi le Prospectus de Diderot. Paris : Vrin.

D'Alembert J. et Diderot D. 1751-1765 (volumes de textes), 1762-1772 (volumes de planches), 1766-1780 (volumes de suppléments et de tables). Encyclopédie ou dictionnaire raisonné des sciences, des arts et des métiers par une société de gens de lettres, Paris : Briasson, David, Le Breton et Durand (vol. de textes et planches), puis Paris : Panckoucke et Amsterdam. Rey (vol. de suppléments et de tables).

Barthes R. [1953] 1972. Le degré zéro de l'écriture. Paris : Seuil.

Canguilhem G. 1965. La connaissance de la vie. Paris : Vrin.

Descartes R. [1897-1909] 1996. Euvres. Paris : Éditions du CNRS et Vrin.

Le Ru V. 2007. Subversives Lumières - L'Encyclopédie comme machine de guerre. Paris : CNRS Éditions.

Montesquieu Ch. 1993. Essai sur le goût, annoté et postfacé par Desgraves L. Paris : Payot et Rivages.

The complete Encyclopedia or how, with the example of astonishment, text and plates can be connected

ABSTRACT: It is a fact: Encyclopedia's text (in 17 volumes) is read without considering the plates (in 11 volumes), but it could be useful and relevant to take an example of a strong interaction between text and plates. The example that has been chosen is a passion: astonishment. To compare its presentation in text and plates, three particular plates from DESSIN are studied and all the articles about the passion of astonishment and surprise are examined.

Keywords: text, plates, dessin, astonishment, surprise. 